\title{
Laparoscopic Approaches to Splenic Flexure Mobilization Tailored According to Disease- and Patient-Related Factors
}

\author{
Melissa Kyriakos Saad ${ }^{1, \odot}$ Elias Saikaly², \\ ${ }^{1}$ General Surgery Department, Colorectal Surgery, Saint George \\ Hospital University Medical Center, Beirut, Lebanon \\ ${ }^{2}$ General Surgery Department, Saint George Hospital University \\ Medical Center, Beirut, Lebanon \\ Int J Recent Surg Med Sci 2021;7:8-12.
}

\begin{abstract}
Early in the 1990s, minimally invasive surgery manifested in laparoscopic surgery found its way to the field of colorectal surgery. Since then, a rising trend in utilizing laparoscopic approach in colorectal surgery, either for benign or malignant disease, is being noticed. In laparoscopic colorectal surgery, the most difficult and challenging step for colorectal surgeons is the mobilization of the splenic flexure. Laparoscopic mobilization of the splenic flexure is an area of debate, with no universally accepted gold standard approach. Multiple approaches have been described in the medical literature and no approach is considered the standard approach. Hence, colorectal sur-

Keywords

- colorectal surgery

- laparoscopic surgery

- splenic flexure approaches geons should be familiar with all the different approaches and they should have the ability of utilizing a tailored splenic flexure mobilization approach modified according to patient- and disease-related factors. Herein, we review the different surgical approaches to laparoscopic splenic flexure mobilization that can be tailored to the surgeons needs according to patient- and disease-related factors.
\end{abstract}

\section{Introduction}

In the medical literature, there is no universal agreement on the definition of splenic flexure mobilization. However, two broad definitions are being utilized based on the attachments being dissected. The partial splenic flexure mobilization includes division of the splenocolic and phrenocolic ligaments, while complete splenic flexure mobilization includes division of the splenocolic, phrenocolic, gastrocolic, and the pancreaticomesocolic attachments.

Splenic flexure mobilization is considered the most difficult, challenging, and demanding step in laparoscopic colorectal surgery. Furthermore, Jamali et al in their questionnaire asked to 35 experienced laparoscopic colorectal surgeons concluded that splenic flexure mobilization is one of the toughest steps in colorectal surgery. ${ }^{1}$ The difficulty in splenic flexure mobilization is related to the complex anatomy of the splenic flexure, and the close proximity of the spleen and pancreas.

Splenic flexure mobilization is deemed mandatory at some instances and elective in others. For example, it is mandatory to mobilize the splenic flexure left mesocolic excision and mainly in splenic flexure colon cancer or while performing total colectomy for ulcerative colitis. However, the main debate lies in the need for routine or selective mobilization of the splenic flexure during left laparoscopic colorectal resections. Currently, there is no universal gold standard on the necessity and advantages of splenic flexure mobilization in left colorectal resections, ${ }^{2,3}$ and colorectal surgeons are divided between proponents and opponents. published online

November 29, 2020
DOI https://doi.org/

$10.1055 / \mathrm{s}-0040-1721539$ ISSN 2455-7420. (c) 2020. Medical and Surgical Update Society.

This is an open access article published by Thieme under the terms of the Creative Commons Attribution-NonDerivative-NonCommercial-License, permitting copying and reproduction so long as the original work is given appropriate credit. Contents may not be used for commercial purposes, or adapted, remixed, transformed or built upon. (https://creativecommons.org/licenses/by-nc-nd/4.0/)

Thieme Medical and Scientific Publishers Pvt. Ltd., A-12, 2nd Floor, Sector 2, Noida-201301 UP, India 
Proponents advocated their support to routine splenic flexure mobilization due to improved postoperative outcome, with some series concluding a better oncologic outcome and lower rate of anastomotic leak in anterior and low anterior resections when implementing routine splenic flexure mobilization. ${ }^{4.5}$ Furthermore, several oncological and anatomical studies favor the routine use of routine splenic flexure mobilization and support its practice. ${ }^{6,7}$ In fact, this is attributed to the extracolonic length gained after deconstructing the splenic flexure, hence reducing the tension on the colorectal anastomosis, preserving the blood supply and consequently a better surgical outcome. Moreover, Aranjo et $\mathrm{al}^{8}$ showed the length of the resected left colon, enabling a tension-free colorectal anastomosis at the level of sacral promontory, is increased by $11 \mathrm{~cm}$ when partial splenic flexure mobilization was performed and $21 \mathrm{~cm}$ when complete splenic flexure mobilization was performed.

In brief, proponents do admit the associated risks of routine splenic flexure mobilization; however, they believe that the morbidity and mortality that result from anastomotic complications prevail over the associated risks. ${ }^{9,10}$

Opponents of routine splenic flexure mobilization argue that splenic flexure mobilization is a complex procedure, not a complication free approach, and time consuming. For instance, Akiyoshi et al reported bleeding near the pancreatic tail and marginal artery injury among the complications associated with splenic flexure mobilization. ${ }^{4}$ Furthermore, the incidence of postoperative complication rate has been documented to be $22.2 \%$ with partial splenic flexure mobilization and $36 \%$ with complete splenic flexure mobilization. ${ }^{12}$ Furthermore, it has been reported that splenic flexure mobilization is associated with longer operative times regardless whether the splenic flexure was approached laparoscopically or by open surgery. 2,4,12

Despite this unsolved debate, most authors believe that splenic flexure mobilization is a complex and challenging step and that proper understanding of the anatomy and embryologic development of the colonic splenic flexure is a must for a good surgical outcome. Furthermore, there is a universal agreement that splenic flexure mobilization is needed at some instances to achieve: an adequate oncologic resection, creation of tension free and well vascularized anastomosis, restoration of the gastrointestinal continuity in rectal resections, and aids in performing a neorectum.

Even with being a fundamental step in colorectal surgery, controversy still exists about the optimal surgical approach, which approach should be the first choice. To date, seven types of laparoscopic splenic flexure mobilization approaches have been described. The aim of this study is to review the different surgical approaches to laparoscopic splenic flexure mobilization tailored to the surgeons needs according to patient- and disease-related factors, starting with a description of the different ligaments that should be deconstructed for complete splenic flexure mobilization to be achieved, moving then to brief description on different approaches and discussing the best approach in each patient and each disease.

\section{Description of Ligaments}

1. The phrenocolic ligament attaches the descending colon and the splenic flexure to the parietal peritoneum ( - Fig. 1).

2. The gastrocolic ligament attaches the transverse colon and the splenic flexure to the greater omentum.

3. The splenocolic ligament attaches the colon to the spleen, also known as the omental bursa (-Fig. 1).

4. The pancreaticomesocolic ligament attaches the transverse mesocolon to the body and tail of the pancreas (-Fig. 1).

\section{Surgical Approach}

\section{The Inferior Approach}

- Cephalic retraction of the transverse colon and medial retraction of the small bowel.

- Identify the ligament of Treitz and the inferior mesenteric vein, and lift the inferior mesenteric vein.

- Dissection of the peritoneum covering the inferior mesenteric vein (-Fig. 2).

- Create a plane bordered anteriorly by the mesocolon and posteriorly by the retro peritoneum.

- Dissection is continued laterally toward the abdominal wall and superiorly till reaching the lower edge of the pancreas and the spleen.

- After finishing these steps, the colon is placed back into the anatomical position and the splenic flexure attachments are tackled.

\section{The Medial-to-Lateral Approach}

- Identify and isolate the inferior mesenteric artery with a T shape, the upper wing directed toward the splenic flexure and the lower wing toward the pelvis.

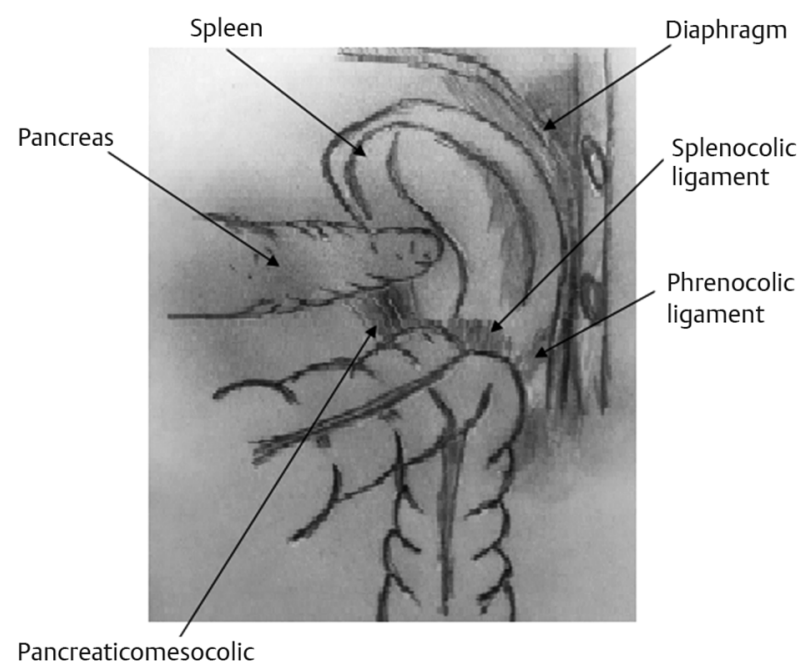

ligament

Fig. 1 Splenic flexure attachments. 
- Start dissection at the level of the inferior mesenteric artery (-Fig. $\mathbf{3}$ ).

- Creation of a plane bordered anteriorly by the mesocolon and posteriorly by the retro peritoneum.

- Dissection continues laterally till reaching the abdominal wall.

- Identification, isolation, and division of the inferior mesenteric vein.

- Colon placed back in anatomical position.

- Division of the remaining lateral attachments moving superiorly to reach the splenocolic ligament that is divided, till reaching the anterior surface of the pancreas.

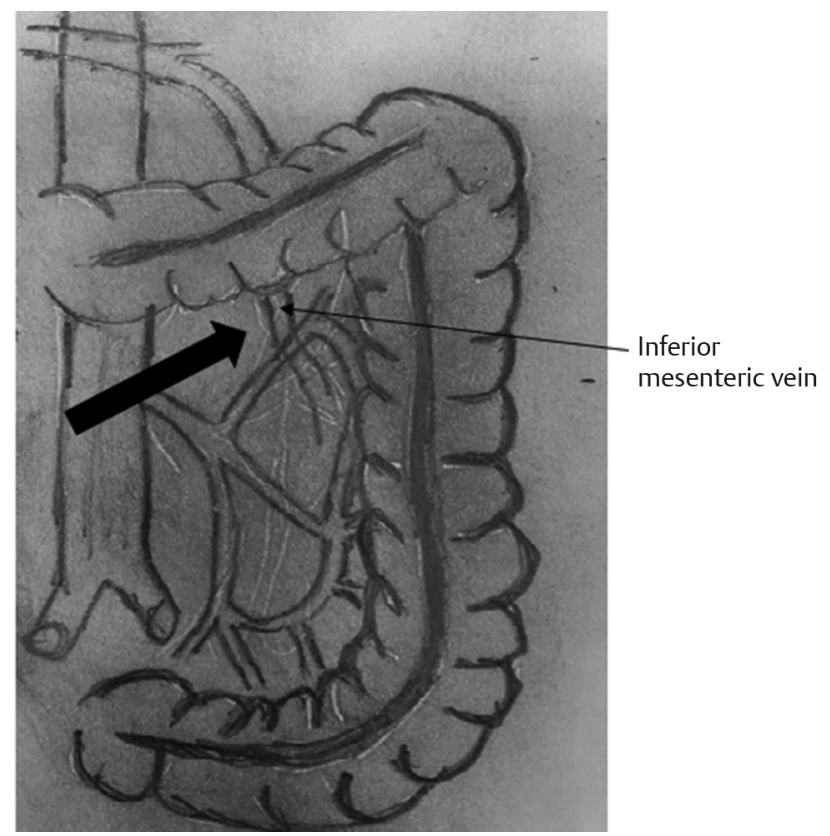

Fig. 2 Inferior approach.

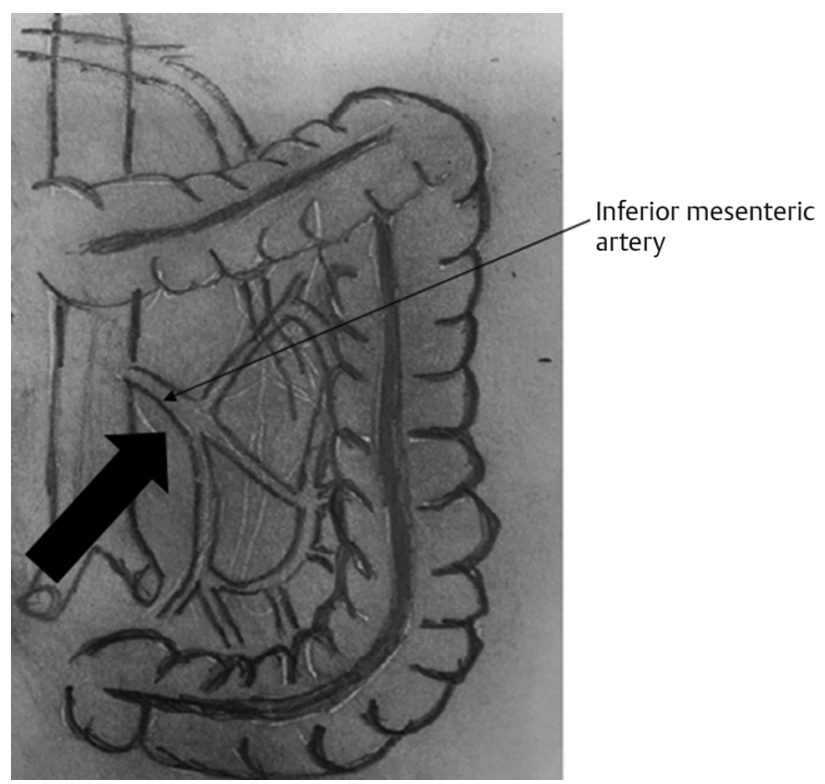

Fig. 3 Medial approach.
- Shifting toward dividing the gastrocolic ligament starting medially and moving laterally till reaching the previously dissected planes.

\section{The Lateral-to-Medial Approach}

- Start with lateral mobilization of the left colon up to the splenic flexure, continue till detaching the colon from Gerota's fascia. It is fundamental to keep "hugging the mesocolon" in this approach (-Fig. 4).

- Continue till reaching the splenocolic ligament that is dissected.

- Carefully free the attachments between the inferior border of the pancreas and the transverse mesocolon.

- Move medially to enter the lesser sac from the right and moving toward the left.

- Continue mobilization laterally toward the splenic flexure till reaching the previously dissected plane.

\section{The Omega Approach: Also Known as the "Rendezvous" Approach}

- The splenic flexure is approached from both sides, alternating the dissection between the right and the left side. This will make evident what should be dissected further (-Fig. 5).

- The omentum is dissected from the distal transverse colon medially moving to the lateral side as the colon is simultaneously retracted both distal and proximal to the flexure inferiorly, showing an "omega" sign.

- Care should be practiced to avoid undue excessive tension on the spleen leading a tear in the splenic capsule.

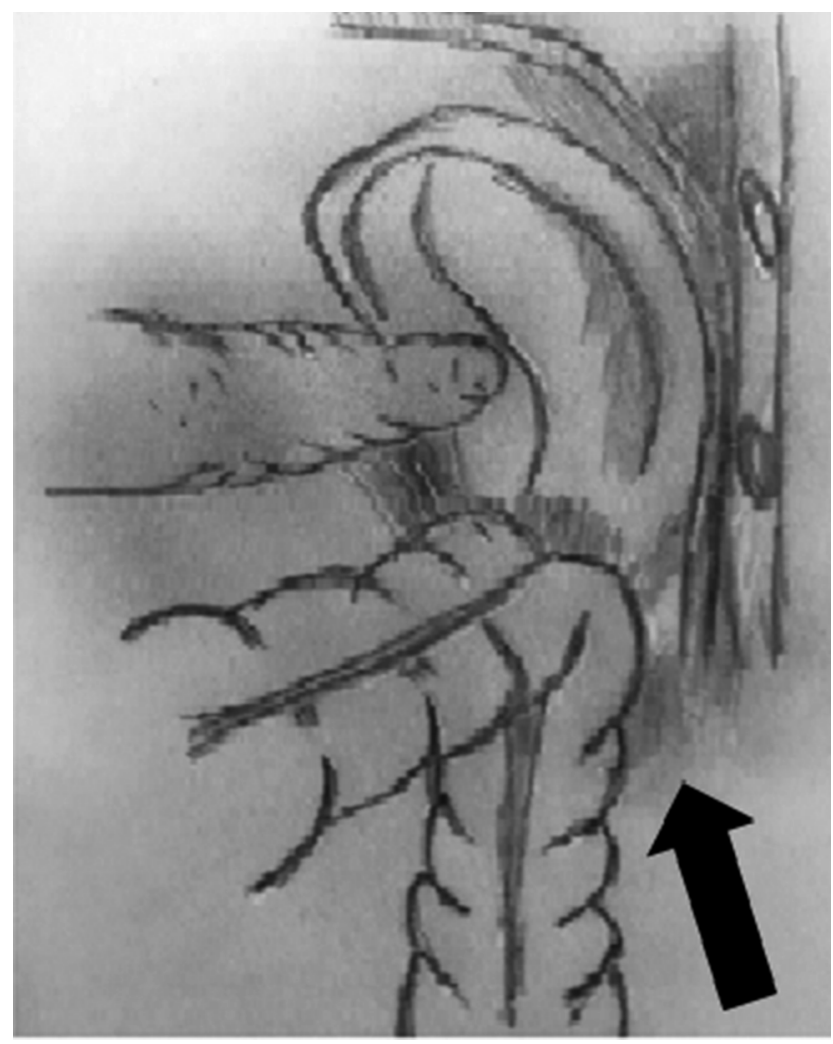

Fig. 4 Lateral approach. 


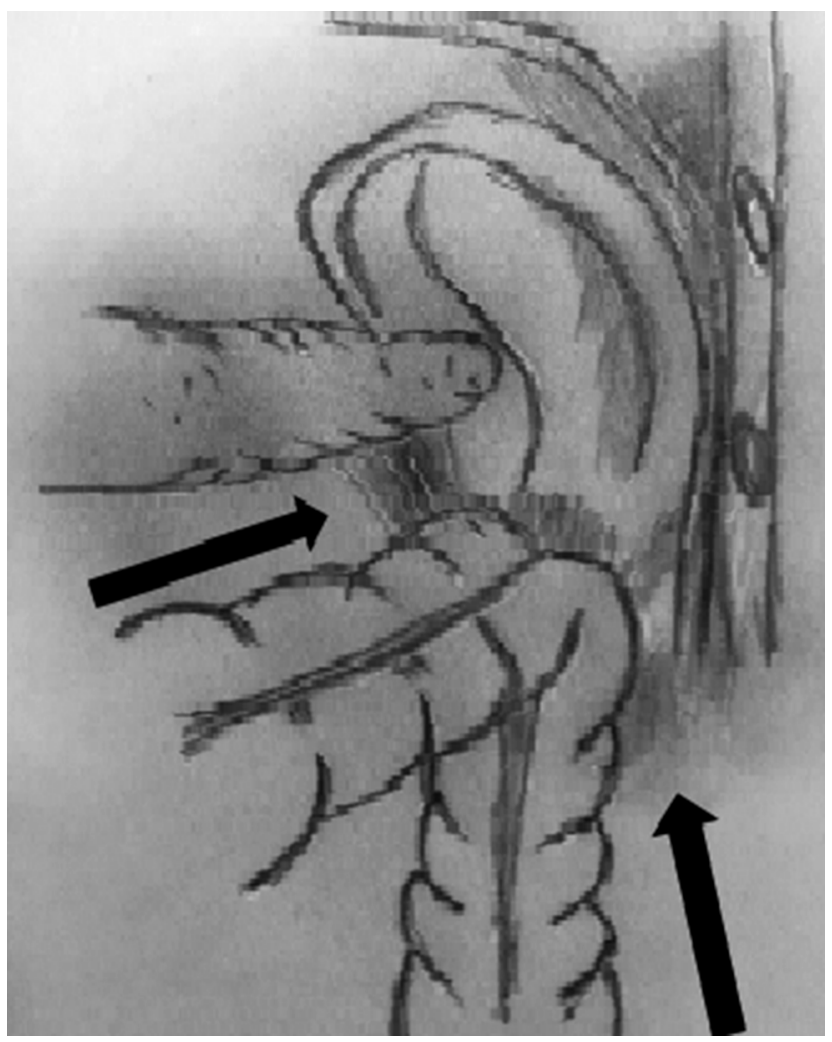

Fig. 5 Omega approach.

\section{Supramesocolic Anterior Approach}

- Start by dissecting the gastrocolic ligament starting medially at the level of the falciform ligament.

- Enter the lesser sac where the posterior border of the stomach and the anterior border of the pancreas will be identified.

- Continue dissection close to the mesocolon till reaching the inferior pole of the spleen.

- Divide the splenocolic ligament.

- Continue dissection of the lateral colonic attachments.

\section{Transomentum Anterior Approach}

- Enter to the lesser sac through dissection into the greater omentum.

- Include part of the greater omentum in the surgical specimen.

- Identify the posterior border of the stomach and the anterior border of the pancreas.

- Continue dissection close to the mesocolon till reaching the inferior pole of the spleen.

- Divide the splenocolic ligament.

- Continue dissection of the lateral colonic attachments.

\section{The Right Lateral Position Approach}

As its name indicates, this approach requires the patient to be placed in the right lateral position.
- Small bowel, stomach, and greater omentum fall away from the descending colon with minimal assistance or retraction.

- Start with dissection of the lateral attachments of the colon.

- Continue dissection to detach the mesocolon from Gerota's fascia.

- At this point, the tail of the pancreas will be identified, as well as the inferior mesenteric vein that will be isolated and divided.

\section{Hand-Assisted Approach or Converting to Hand-Assisted Approach}

Usually an additive to the above-mentioned approaches, additional retraction can be achieved and hence better visualization.

\section{Compound Approach}

Combine the above-mentioned different approaches, moving back and forth from one approach to another and back to another aids in mobilizing the splenic flexure.

\section{Discussion}

As the surgical technique is considered as independent prognostic factor in the oncologic surgery outcome, in-depth knowledge of the anatomy, and hence surgical approach, is essential. Splenic flexure mobilization is considered the most difficult, challenging, and demanding maneuver in laparoscopic colorectal surgery. The learning curve for splenic flexure mobilization is slow. This is attributed to the rarity of splenic flexure colon cancer as compared with other colon cancers, adding to this the complex anatomy of the colonic splenic flexure and the possibility of iatrogenic injury to the spleen and pancreas and its associated morbidity. ${ }^{13}$

In view the debate surrounding the selective versus routine splenic flexure mobilization for left colorectal resections, colorectal surgeons are being divided into opponents and proponents. The main challenge lies in identification of the best approach to deconstruct the splenic flexure taking into account disease- and patient-related factors. Hence, splenic flexure mobilization should be tailored according to the patient and the underlying disease. For instance, in patients with previous history of recurrent pancreatitis the medial and inferior approach should be avoided due to the risk of injury to the marginal arcade. ${ }^{14}$ The latter mentioned approaches should also be avoided or discouraged for obese patients, due to the difficulties encountered in identifying the body and tail of the pancreas due to increased levels of adipose tissue, adding to this the risk of injury to the marginal arcade in obese patients..$^{15}$ Furthermore, in patients with splenic flexure colon cancer, it is advised to use the transomentum anterior approach as splenic flexure colon cancer presents an incidence of omental implants of up to $15 \%$. Furthermore, they present an incidence of affected 
lymph nodes at the level of gastroepiploic arcade in up to $25 \%$ of cases; hence, these lymph nodes should also be included in the specimen. ${ }^{16}$ However, the transomentum anterior approach should be avoided in low anterior or anterior resections, as this detached omentum will eventually be necrotic leading to abscess formation. Having said this and knowing that splenic flexure mobilization is a fundamental step in colorectal surgery, controversy still exists about the optimal surgical approach, which should be the first choice. However, knowledge of all the possible approaches is essential and in the difficult splenic flexure a combination of these approaches is deemed necessary. In brief, a tailored splenic flexure mobilization approach modified according to patient-related and disease-related factors is the key for a good surgical outcome.

\section{Conclusion}

To date, there is no approach to laparoscopic splenic flexure mobilization that is considered the gold standard surgical approach. The choice among the different surgical approaches should be tailored according to patient-related factors such as obesity and pancreatitis and disease-related factors such as splenic flexure colon cancer and low rectal cancer. However, in-depth knowledge of this complex anatomy and different surgical approaches is a must for every colorectal surgeon.

\section{Conflict of Interest}

None declared.

\section{References}

1 Jamali FR, Soweid AM, Dimassi H, Bailey C, Leroy J, Marescaux J. Evaluating the degree of difficulty of laparoscopic colorectal surgery. Arch Surg 2008;143(8):762-767, discussion 768

2 Brennan DJ, Moynagh M, Brannigan AE, Gleeson F, Rowland M, O'Connell PR. Routine mobilization of the splenic flexure is not necessary during anterior resection for rectal cancer. Dis Colon Rectum 2007;50(3):302-307, discussion 307

3 Katory M, Tang CL, Koh WL, et al. A 6-year review of surgical morbidity and oncological outcome after high anterior resection for colorectal malignancy with and without splenic flexure mobilization. Colorectal Dis 2008;10(2):165-169

4 Akiyoshi T, Kuroyanagi H, Oya M, et al. Factors affecting difficulty of laparoscopic surgery for left-sided colon cancer. Surg Endosc 2010;24(11):2749-2754

5 Farke S, Bouchard R, Blumberg C, et al. Mobilization of the splenic flexure: a standard in laparoscopic left colon and rectum resections! Surg J 2010;5:31-35

6 Araujo SE, Seid VE, Kim NJ, Bertoncini AB, Nahas SC, Cecconello I. Assessing the extent of colon lengthening due to splenic flexure mobilization techniques: a cadaver study. Arq Gastroenterol 2012;49(3):219-222

7 Kye BH, Kim HJ, Kim HS, Kim JG, Cho HM. How much colonic redundancy could be obtained by splenic flexure mobilization in laparoscopic anterior or low anterior resection? Int J Med Sci 2014;11(9):857-862

8 Araujo SEA, Seid VE, Kim NJ, Bertoncini AB, Nahas SC, Cecconello I. Assessing the extent of colon lengthening due to splenic flexure mobilization techniques: a cadaver study. Arquivos de gastroenterologia. 2012;49:219-222

9 Ludwig KA, Kosinski L. Is splenic flexure mobilization necessary in laparoscopic anterior resection? Another view. Dis Colon Rectum 2012;55(11):1198-1200

10 Taflampas P, Christodoulakis M, Tsiftsis DD. Anastomotic leakage after low anterior resection for rectal cancer: facts, obscurity, and fiction. Surg Today 2009;39(3):183-188

11 Gezen C, Altuntas YE, Kement M, et al. Complete versus partial mobilization of splenic flexure during laparoscopic low anterior resection for rectal tumors: a comparative study. J Laparoendosc Adv Surg Tech A 2012;22(4):392-396

12 Marsden MR, ContiJA, Zeidan S, et al. The selective use of splenic flexure mobilization is safe in both laparoscopic and open anterior resections. Colorectal Dis 2012;14(10):1255-1261

13 Saber AA, Dervishaj O, Aida SS, Christos PJ, Dakhel M. CT Scan mapping of splenic flexure in relation to spleen and its clinical implications. Am Surg 2016;82(5):416-419

14 Perrakis A, Weber K, Merkel S, et al. Lymph node metastasis of carcinomas of transverse colon including flexures. Consideration of the extramesocolic lymph node stations. Int J Colorectal Dis 2014;29(10):1223-1229

15 Kim HJ, Kim CH, Lim SW, Huh JW, Kim YJ, Kim HR. An extended medial to lateral approach to mobilize the splenic flexure during laparoscopic low anterior resection. Colorectal Dis 2013;15(2):e93-e98

16 Watanabe J, Ota M, Suwa Y, Ishibe A, Masui H, Nagahori K. Evaluation of lymph flow patterns in splenic flexural colon cancers using laparoscopic real-time indocyanine green fluorescence imaging. Int J Colorectal Dis 2017;32(2):201-207 